\title{
No evidence of XMRV in prostate cancer cohorts in the Midwestern United States
}

\author{
Toshie Sakuma', Stéphane Hué2, Karen A Squillace', Jason M Tonne', Patrick R Blackburn', Seiga Ohmine',
} Tayaramma Thatava ${ }^{1}$, Greg J Towers ${ }^{2}$ and Yasuhiro Ikeda ${ }^{*}$

\begin{abstract}
Background: Xenotropic murine leukemia virus (MLV)-related virus (XMRV) was initially identified in prostate cancer (PCa) tissue, particularly in the prostatic stromal fibroblasts, of patients homozygous for the RNASEL R462Q mutation. A subsequent study reported XMRV antigens in malignant prostatic epithelium and association of XMRV infection with PCa, especially higher-grade tumors, independently of the RNASEL polymorphism. Further studies showed high prevalence of XMRV or related MLV sequences in chronic fatigue syndrome patients (CFS), while others found no, or low, prevalence of XMRV in a variety of diseases including PCa or CFS. Thus, the etiological link between XMRV and human disease remains elusive. To address the association between XMRV infection and PCa, we have tested prostate tissues and human sera for the presence of viral DNA, viral antigens and anti-XMRV antibodies.
\end{abstract}

Results: Real-time PCR analysis of 110 PCa (Gleason scores >4) and 40 benign and normal prostate tissues identified six positive samples (5 PCa and 1 non-PCa). No statistical link was observed between the presence of proviral DNA and PCa, PCa grades, and the RNASEL R462Q mutation. The amplified viral sequences were distantly related to XMRV, but nearly identical to endogenous MLV sequences in mice. The PCR positive samples were also positive for mouse mitochondrial DNA by nested PCR, suggesting contamination of the samples with mouse DNA. Immuno-histochemistry (IHC) with an anti-XMRV antibody, but not an anti-MLV antibody that recognizes XMRV, sporadically identified antigen-positive cells in prostatic epithelium, irrespectively of the status of viral DNA detection. No serum (159 PCa and 201 age-matched controls) showed strong neutralization of XMRV infection at 1:10 dilution.

Conclusion: The lack of XMRV sequences or strong anti-XMRV neutralizing antibodies indicates no or very low prevalence of XMRV in our cohorts. We conclude that real-time PCR- and IHC-positive samples were due to laboratory contamination and non-specific immune reactions, respectively.

\section{Background}

Prostate cancer (PCa) is the most frequently diagnosed noncutaneous malignancy among men in industrialized countries [1]. Although early detection using tests for prostate-specific antigen and improved treatment have emerged as important interventions for decreasing PCa mortality, there is potential for improved prognosis through detection of genetic risk factors. Indeed, a positive family history is among the strongest epidemiological risk factors for $\mathrm{PCa}$, and a number of genetic mutations have been implicated in PCa. For example, an

\footnotetext{
* Correspondence: ikeda.yasuhiro@mayo.edu

'Department of Molecular Medicine, Mayo Clinic, Rochester, MN 55905 USA Full list of author information is available at the end of the article
}

R462Q polymorphism in the RNase L protein, which impairs the catalytic activity of an important effector of the innate antiviral response, has been implicated in up to $13 \%$ of unselected PCa cases [2].

Xenotropic murine leukemia virus (MLV)-related virus (XMRV) was first identified in PCa tissues, particularly those with the homozygous RNASEL R462Q mutation [3]. Genetic analysis identified XMRV as a xenotropic gammaretrovirus, closely related to those found in mice $[4,5]$. This suggested that XMRV represented a zoonotic transmission from mice to humans. When compared with exogenous and endogenous MLV sequences, XMRV appeared to have a unique, conserved $24 \mathrm{bp}$ deletion in the gag leader region [3]. However, this

\section{Biomed Central}


deletion has recently been found in endogenous MLV proviruses in a variety of mice [6]. Initially, immuno-histochemistry (IHC) and FISH analyses suggested that only prostatic stromal fibroblasts were infected with XMRV [3]. Subsequently, Schlaberg, Singh and colleagues reported the expression of XMRV antigens in 23\% of PCa and an association of XMRV infection with higher grade tumors [7]. Contrary to the initial study, Singh's study found viral antigen-positive cells primarily in malignant prostatic epithelium, independently of the RNASEL polymorphism [7]. It is notable that this study found many immuno-histochemistry-positive samples which did not have detectable XMRV DNA [7]. Another study found 11 (27.5\%) of 40 PCa patients with XMRV neutralizing antibodies [8]. Importantly, there were correlations between serum positivity and nested PCR results, FISH, or the R462Q RNASEL mutation [8]. In sharp contrast, several recent reports found no or very low prevalence of XMRV (DNA, RNA or antibodies) in PCa samples [9-12].

If the role of XMRV in PCa is confirmed, detection and prevention of XMRV infection could provide a novel intervention strategy for early diagnosis and treatment of PCa. However, the conflicting epidemiological data have made it unclear whether XMRV plays a role in PCa and have questioned whether the virus is truly a human pathogen. In this study we have sought to address the association between XMRV infection and PCa, PCa grades and RNASEL R462Q polymorphism by testing prostate tissues for the presence of XMRV. In addition, to determine the correlation between $\mathrm{PCa}$ and seroprevalence of XMRV, serum samples from patients with $\mathrm{PCa}$ were compared with age-matched controls for detectable anti-XMRV antibodies. Our study found no XMRV sequences and no XMRV-neutralizing antibodies in 150 prostate tissues (110 $\mathrm{PCa}$ and 40 benign/normal) and serum samples (159 PCa and 201 age-matched controls), respectively, indicating no or very low prevalence of XMRV in our cohorts. We did detect MLV sequences in 6 samples, but these samples were also PCR positive for mouse mitochondrial DNA suggesting DNA contamination as a source of the MLV. We were therefore unable to confirm the links between XMRV infection with $\mathrm{PCa}, \mathrm{PCa}$ grades or RNASEL mutation.

\section{Results}

Prevalence of XMRV proviral DNA in PCa

We have previously developed a real-time PCR assay for detection of XMRV gag sequences $[13,14]$. Tests using the XMRV infectious molecular clone plasmid, pcDNA3.1(-)/VP62, could detect a single copy of the XMRV genome in $1.0 \mu \mathrm{g}$ of total cellular DNA (approximately $1.4 \times 10^{5}$ cells). The primers and the probe used in this assay were designed to detect most MLV-related sequences from mice. Using this sensitive real-time PCR assay, we screened DNA from 150 prostate tissues (110 $\mathrm{PCa}$ and $40 \mathrm{benign/normal} \mathrm{controls).} \mathrm{One} \mathrm{out} \mathrm{of} 40$ high grade PCa (Gleason score 8-10), 4 out of 70 intermediate grade PCa (Gleason score 5-7), and 1 out of 40 benign/normal prostate tissues (Gleason score $<4$ ) were repeatedly positive by this assay (Table 1 ). The viral DNA copy numbers ranged from 0.5 to 11 copies per $1.0 \mu \mathrm{g}$ DNA (average of 4 reactions). As one diploid cell contains approximately $7.1 \mathrm{pg}$ of DNA, we estimate that PCR-positive clinical samples had 0.5 to 11 copies of proviral DNA in $1.4 \times 10^{5}$ cells.

To confirm the real-time PCR results, we screened the same DNA samples by nested PCR for XMRV/MLV gag sequences. In order to establish consistency and to minimize the risk of contamination during the procedure, three individuals independently performed the nested PCR experiments using independently aliquoted DNA samples. Four out of 6 real-time PCR positive samples (\#15, 51, 52 and 112) were consistently positive by the nested PCR analysis, while the other two positive samples from intermediate grade PCa (\#53 and 103) were shown to be nested PCR-positive twice in the first three attempts. Further analysis confirmed that these two samples were nested PCR-positive for viral DNA. The 144 real-time PCR-negative samples were also found to be negative by nested PCR.

\section{No statistical link between the presence of viral DNA and} prostate cancer or higher tumor grade

We then sought a correlation between viral DNA detection and the presence of PCa. There was no statistical difference between the frequency of PCR positivity in $\mathrm{PCa}$ and in benign/normal controls (Table 2). We also examined a link between PCR positivity and tumor grade as measured by the Gleason score. Using total of 110 DNA samples from $\mathrm{PCa}, 4$ out of 70 intermediate grade (Gleason score 5-7) and 1 out of 40 high grade (Gleason score 8-10) were positive by real time PCR (Table 3). These data were not statistically significant by

Table 1 Prevalence of XMRV and tumor grade

\begin{tabular}{lcc}
\hline & No. $^{a}$ & Positive $^{\boldsymbol{b}}$ \\
\hline Low grade & & \\
Gleason 5 & 40 & 1 \\
Gleason 6 & 2 & 0 \\
Gleason 7 & 22 & 3 \\
Gleason 8 & 46 & 1 \\
Gleason 9 & 16 & 1 \\
Gleason 10 & 23 & 0 \\
\hline
\end{tabular}

${ }^{a}$ Total number of samples tested from each Gleason score.

${ }^{b}$ Number of PCR positive samples from each Gleason score.

'Low grade includes Gleason score 1 through 4. 
Table 2 Statistical analysis of XMRV positivity in controls and $\mathrm{PCa}$

\begin{tabular}{lccc}
\hline & No. $^{a}$ & Positive $^{b}$ & $\boldsymbol{x}^{\mathbf{c}}$ \\
\hline $\mathrm{Non}^{\mathrm{P}} \mathrm{PCa}^{d}$ & 40 & 1 & 0 \\
$\mathrm{PCa}^{e}$ & 110 & 5 & 0.319 \\
\hline
\end{tabular}

${ }^{a}$ Total number of samples tested from each Gleason score.

${ }^{b}$ Number of PCR positive samples from each Gleason score.

'Statistical results from chi-square $\left(x^{2}\right)$ tests.

${ }^{d}$ Samples from benign/normal cancer patients.

${ }^{e}$ Samples from prostate cancer patients.

chi-square $\left(x^{2}\right)$ as indicated in Table 3, suggesting no correlation between the prevalence of viral DNA and higher tumor grade in our samples.

\section{No correlation between viral DNA detection and RNASEL R462Q mutation}

In order to consider the association between RNASEL mutation and viral infection, we amplified part of the RNASEL gene by PCR and determined the status of the R462Q RNASEL polymorphism. Of 150 prostate tissues, 20 cases were found to be homozygous for RNASEL R462Q (Table 4). However, these samples were all negative for viral DNA by real-time PCR. Thus there was no linkage between viral DNA detection and RNASEL R462Q in our clinical samples (Table 5).

\section{Phylogenetic analyses of MLV-like sequences in prostate tissue DNA}

XMRV has been PCR amplified from prostate cancer samples in a number of studies $[3,8,12]$ as well as in blood samples from patients with chronic fatigue syndrome (CFS) [15]. Furthermore, a recent study reported a high frequency of MLV that was distinct from XMRV by PCR in patients with chronic fatigue syndrome [16]. To examine the viral sequences identified in our $\mathrm{PCa}$ samples, we cloned the PCR-amplified DNA bands from four viral DNA-positive patient samples (\#15 [GenBank no. JF288880, JF288881], \#51 [GenBank no. JF288878, JF288879], \#52 [GenBank no. JF288882, JF288883] and \#112 [GenBank no. JF288884]) and determined their nucleotide sequences. We were able to identify two independent sequences from each of patients \#15, \#51 and $\# 52$ and a single sequence from patient \#112. To

Table 3 Statistical analysis of XMRV prevalence and tumor grade

\begin{tabular}{lccc}
\hline & No. $^{\boldsymbol{a}}$ & Positive $^{\boldsymbol{b}}$ & $\boldsymbol{x}^{2 \boldsymbol{c}}$ \\
\hline Low grade & 40 & 1 & 0 \\
Intermediate & 70 & 4 & 0.606 \\
High grade & 40 & 1 & 0 \\
\hline
\end{tabular}

${ }^{a}$ Total number of samples tested from each Gleason score.

${ }^{b}$ Number of PCR positive samples from each Gleason score.

${ }^{c}$ Statistical results from chi-square $\left(x^{2}\right)$ tests.
Table 4 RNASEL genotyping and tumor grade

\begin{tabular}{lcccc}
\hline & Normal/benign $^{\boldsymbol{a}}$ & Intermediate $^{\boldsymbol{b}}$ & High $^{\boldsymbol{c}}$ & Total $^{\boldsymbol{d}}$ \\
\hline RNASEL RR & 11 & 28 & 15 & 54 \\
RNASEL RQ & 21 & 36 & 19 & 76 \\
RNASEL QQ & 8 & 6 & 6 & 20 \\
\hline
\end{tabular}

${ }^{a}$ Samples with Gleason score 1 through 4 .

${ }^{b}$ Samples with intermediate Gleason score.

'Samples with high Gleason score.

${ }^{d}$ Total numbers of each RNASEL genotypes.

compare these sequences to XMRV and to previously published MLV sequences from mice and patient samples, we reconstructed Bayesian phylogenies (Figure 1). None of the gag gene sequences amplified from our clinical samples belonged to the clade formed by previously reported XMRV sequences; instead, they clustered with known polytropic murine leukemia virus (PMLV), modified polytropic murine leukemia virus (MPMLV) or xenotropic murine leukemia virus (MLV$\mathrm{X}$ ) endogenous sequences of mice (Figure 1). Importantly, one of the patients (\#52) appeared to be infected with two independent MLVs, one from the modified polytropic MLV clade and one from the xenotropic MLV clade. A similar result was seen when a maximum likelihood phylogeny was constructed using the software RAxML [17] (not shown). In each case, BLAST analysis of the amplified sequences identified at least one endogenous MLV sequence in the mouse genome with very high (>99\%) similarity (Table 6). Two of the five fragments were identical to known endogenous proviruses and the other three were greater than 99\% similar. These proviruses exist in multiple locations within the mouse genome.

Because the sequences we amplified were similar to the MLV sequences detected in CFS patients [16], we also analyzed the sequences reported in that study. The sequences amplified from CFS patients also fell into both polytropic and modified polytropic clades of endogenous MLVs (Figure 1). They were also very similar (98-100\%) to known endogenous MLV proviruses in mice (Table 7). In fact, the differences between the amplified sequences and the endogenous sequences are consistent with known error rates of Taq polymerase or could also be explained by polymorphisms between mice [18-20].

Table 5 Statistical analysis of XMRV prevalence and RNASEL genotyping

\begin{tabular}{lccc}
\hline & XMRV $^{a}$ & XMRV $^{\boldsymbol{a}}$ & $\boldsymbol{x}^{\boldsymbol{a}^{\boldsymbol{c}}}$ \\
\hline RNASEL RR+RQ & 6 & 124 & 0 \\
RNASEL QQ & 0 & 20 & 0.962 \\
\hline
\end{tabular}

${ }^{a}$ XMRV positive samples from real time PCR.

${ }^{b}$ XMRV negative samples from real time PCR.

${ }^{c}$ Statistical results from chi-square $\left(x^{2}\right)$ tests. 


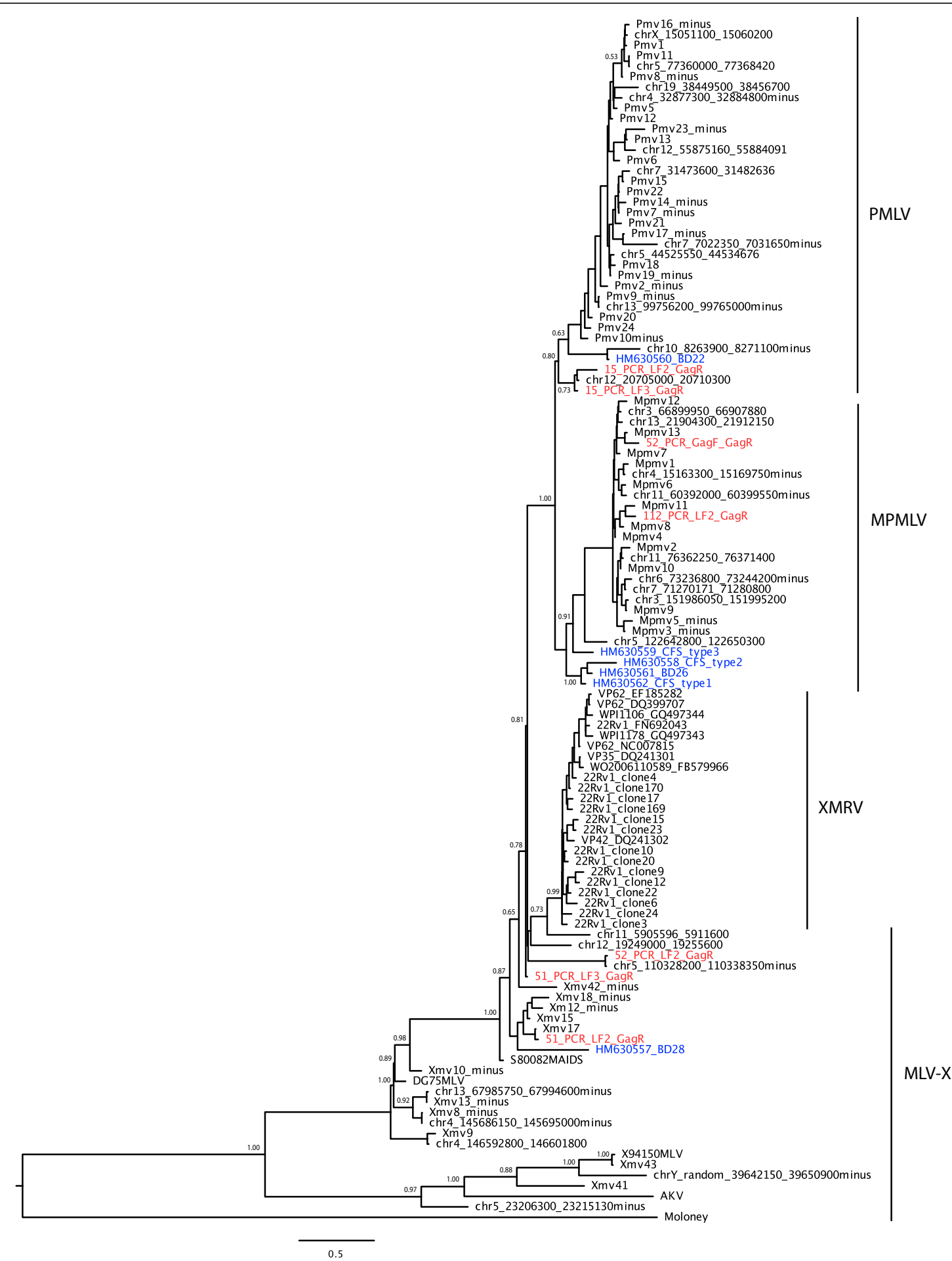

Figure 1 Bayesian maximum clade credibility phylogeny of endogenous murine MLV sequences, $22 \mathrm{Rv} 1$ cell line and patient derived MLV gag gene sequences. Sequences derived from PCa samples in this study are colored red. Sequences from [16] are colored blue. The tree is rooted against the Moloney MLV sequence. Bayesian posterior probabilities above 0.50 are indicated on the corresponding branches. The scale bar represents the number of nucleotide substitutions per site. 
Table 6 Comparison of MLV sequences amplified from patient samples with mouse genomic sequences

\begin{tabular}{ccllll}
\hline Sequence (GenBank no.) & Length (nt) & \multicolumn{1}{c}{ Closest relative } & GenBank no. & Similarity & Nucleotide difference \\
\hline 51_PCR_LF2_GagR (JF288878) & 608 & Mus musculus BAC clone RP23-457E5 & AC121813 & $100 \%$ & $0 / 608$ \\
51_PCR_LF3_GagR (JF288879) & 250 & Mus musculus chrom 7, clone RP24-220N8 & AC167466 & $99 \%$ & $1 / 250$ \\
15_PCR_LF2_GagR (JF288880) & 608 & Mus musculus BAC clone RP23-152O2 & AC163634 & $100 \%$ & $0 / 608$ \\
15_PCR_LF3_GagR (JF288881) & 271 & Mus musculus BAC clone RP23-152O2 & AC163634 & $>99 \%$ & $1 / 271$ \\
52_PCR_GagF_GagR (JF288882) & 525 & Mouse DNA sequence, clone CH29-187G15 & CU407131 & $100 \%$ & $0 / 525$ \\
52_PCR_LF2_GagR (JF288883) & 540 & Mus musculus chrom 5, clone RP23-280N22 & AC123679 & $>99 \%$ & $1 / 540$ \\
112_PCR_LF2_GagR (JF288884) & 691 & Mouse DNA sequence, CH29-187G15 & CU407131 & $>99 \%$ & $6 / 691$ \\
\hline
\end{tabular}

NB: Gaps are treated as mismatches.

The similarity between the patient amplified sequences and known endogenous MLV provirus sequence in mice suggests that the MLVs may have been amplified from samples that had been inadvertently contaminated with mouse DNA. To examine this possibility further in our samples we tested each positive PCa sample for the presence of mouse mitochondrial DNA by PCR. Strikingly, all of the clinical samples that were positive for MLV were also positive for mouse mitochondrial DNA (Figure 2). When the amplified DNA fragments were cloned and sequenced, they were $100 \%$ identical to Mus musculus cytochrome $\mathrm{b}$ gene sequence. Thus, the MLV sequences detected by sensitive PCR methods in patient samples likely originated from contaminating mouse DNA encoding endogenous MLV proviruses.

\section{Detection of XMRV antigens in PCa tissues}

Previous IHC studies found XMRV antigen-positive cells in prostatic stromal fibroblasts [3] or in malignant prostatic epithelium [7]. Importantly, Schlaberg, Singh and colleagues also showed frequent detection of viral antigen-positive cells in PCR-negative tissues [7]. In order to seek viral antigen-positive cells in our clinical samples, we prepared prostate tissue sections and performed IHC analysis. We used the rabbit anti-XMRV antibody, which was used in the previous study by Schlaberg et al. [7]. We also used a goat anti-MLV p30/gp70 antibody, which can detect XMRV precursor Gag, CA, and Env proteins in XMRV transfected cells $[13,14]$. Both antisera showed clear and reproducible staining of 293T cells transfected with the infectious XMRV clone VP62 (Figure 3A) or XMRV-producing 22Rv1 cells (data not shown). No specific staining was seen when uninfected control 293T cells were stained with these antisera (Figure 3A). We prepared tissue sections of four PCRpositive tissues (Gleason scores 6 and 8 ) as well as two PCR negative tissues (Gleason scores 6 and 8, real-time/ nested PCR-double negative), and analyzed them with the two antisera. The anti-XMRV antibody sporadically detected antigen-positive cells, exclusively in prostatic epithelium, in the sections of tissues (Figure 3B, upper middle panel with FITC). Similar results were observed with a different secondary antibody conjugated with Texas Red (Figure 3C). In contrast, no signal was detected with the anti-MLV p30/gp70 in any of the tissue sections (Figure 3B). Importantly, the anti-MLV serum did not stain the cells, which were shown to be IHC-positive by the anti-XMRV serum, in the serial sections of the same tissue (Figure 3B, upper panels). It was also notable that the anti-XMRV serum found antigenpositive cells in PCR-negative tissue sections (Figure 3C), suggesting that this serum also recognizes a non-viral protein. Similar results were recently reported by Switzer et al [21]. Considering the data obtained using the antiMLV serum, we conclude that we cannot detect XMRV in prostate cancer tissues and that the antibody described by Schlaberg, Singh and colleagues recognizes non-viral proteins in addition to XMRV.

Table 7 Comparison of MLV sequences amplified from patient samples [16] with mouse genomic sequences

\begin{tabular}{|c|c|c|c|c|c|}
\hline Sequence & Length (nt) & Closest relative & GenBank no. & Similarity & Nucleotide difference \\
\hline HM630557 & 319 & Mouse endogenous retrovirus & M26006 & $99 \%$ & $4 / 310$ \\
\hline HM630558 & 698 & Mus musculus BAC clone RP23-115O21 & AC163617 & $99 \%$ & $7 / 698$ \\
\hline HM630559 & 698 & Mouse DNA sequence from clone RP23-131N18 & AL772224 & $99 \%$ & $1 / 697$ \\
\hline HM630560 & 697 & Mouse endogenous retrovirus & M26005 & $99 \%$ & $3 / 698$ \\
\hline HM630561 & 339 & Mouse DNA sequence, clone CH29-187G15 & CU407131 & $99 \%$ & $6 / 339$ \\
\hline HM630562 & 698 & Mus musculus BAC clone RP23-115021 & AC163617 & $99 \%$ & $5 / 697$ \\
\hline
\end{tabular}

Patient amplified MLV sequences were used as a BLAST query to identify their closest relative. The accession numbers of the mouse genomic sequences identified are shown as are the number of nucleotide differences between the patient amplified sequence and their nearest relatives in the mouse genome. 


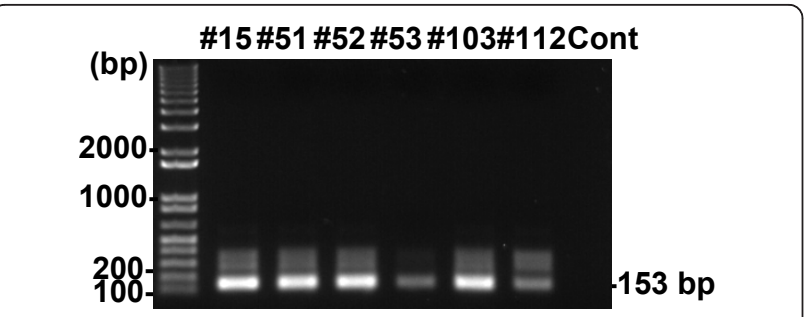

Figure 2 PCR for mouse mitochondrial DNA. $q P C R$ positive samples (\#15, 51, 52, 53, 103, 112) were PCR amplified for mouse mitochondrial DNA. Positive samples yielded PCR products at 153 bp [16]. Water was used as a control.

\section{Absence of XMRV antibodies in patients with PCa and age-matched controls}

Serological testing was performed with a recently developed XMRV neutralizing assay which measures viral neutralizing activity using a GFP-encoding XMRV and flow cytometry [14]. Positive seroreactivity was defined as $100 \%$ block of XMRV-GFP transduction with a 10 -fold diluted serum sample. We randomly sampled 159 PCa cases out of 933 patients who are consenting, age 50-70 and have a clinical Gleason Score of 6 or 7 (most common) in the Mayo Clinic Prostate SPORE Biospecimen files. 201 sera from age-matched patients without $\mathrm{PCa}$ or any known urological disorders were included as non-PCa controls. As positive controls, we used anti-XMRV sera from XMRV-infected wild mice, Mus pahari [14]. Sera from XMRV-infected mice diluted 10-fold completely blocked XMRV-GFP infection (Figure 4A). In contrast, none of the clinical samples showed strong anti-XMRV activity at 10-fold dilution (Figure 4B). Two out of 159 PCa (Figure 4C) and five out of 201 non-PCa (Figure 4D) sera marginally reduced the XMRV infectivity (over $80 \%$ block of XMRV infectivity at a 10 -fold dilution). However, by Western blot probing cell lysates from XMRV-infected and uninfected cells, these patients' sera failed to detect XMRV Env, Gag or p30 Capsid (data not shown). To rule out the possibility that these patients' sera cannot detect denatured XMRV proteins by Western blotting, we also performed the indirect immunofluorescent assay using HeLa cells (control) and XMRV-infected HeLa cells as antigens. None of the sera could detect XMRV antigens in HeLa cells at 50- and 200-fold dilutions (data not shown). We, therefore, conclude that XMRV antibodies are absent from our patient population.

\section{Discussion}

In this study, we have examined the prevalence of XMRV in patients with or without PCa at Mayo Clinic. We were unable to find XMRV sequences or antiXMRV antibodies in our patients, most of whom are from the mid-west area of the USA, indicating that there is no or very low prevalence of XMRV in this region. Moreover, we were unable to confirm the correlation between XMRV infection and $\mathrm{PCa}$, higher tumor grade or RNASEL R462Q mutation.

A high prevalence of XMRV has been reported in patients with $\mathrm{PCa}$ and chronic fatigue syndrome (CFS) in the USA $[3,7,8]$, but similar studies in Europe have failed to detect XMRV [10-12]. It has been suggested that geographical differences might explain this striking variation in XMRV prevalence [11] but our results, as well as recent US studies that also find no evidence for XMRV $[9,21]$, appear to rule this explanation out. In this regard, it is notable that previous studies to identify XMRV in patients with PCa or chronic fatigue syndrome have relied on very sensitive PCR detection methods. Because of the high similarity between patient associated XMRV/MLV and endogenous MLV sequences and the striking discordance between studies, it has been suggested that PCR-positive results might be attributed to unintentional detection of contaminating mouse DNA in human specimens [6,22-24]. It is notable that Lo et al. [16] detected polytropic and modified polytropic MLV sequences, but not XMRV, in blood samples from chronic fatigue patients (Figure 1). These authors were unable to identify the samples as contaminated using mouse mitochondrial PCR. In our study, real-time PCR and nested PCR identified 6 of 150 samples as positive for MLV. However, the amplified sequences were closely related to known endogenous MLV proviruses, rather than XMRV. In fact one patient sample (\#52) contained two independent MLV sequences. This might be interpreted as evidence for evolution of the virus in the patient but closer analysis reveals that one of the sequences is identical to a known endogenous modified polytropic sequence whilst the other is a single nucleotide different from a known mouse endogenous xenotropic MLV. This, therefore, suggests either infection of this patient with two independent MLVs or PCR contamination with mouse DNA as a source. As all of the MLV PCR-positive samples contained detectable levels of mouse mitochondrial DNA, we conclude that the amplified sequences originated from mouse DNA that somehow contaminated the study samples.

In order to confirm that the viral sequences were amplified from endogenous MLV in mouse genomic DNA, but not replicating MLV in human tissue, we attempted to determine viral integration sites. We first used the protocol described by Kim et al. [25] but failed to amplify DNA sequences containing the partial XMRV LTR. We then designed universal primers to recognize LTRs from XMRV and endogenous and exogenous MLVs [26], as well as a series of primers specific for the viral sequences identified in our clinical samples. 
A

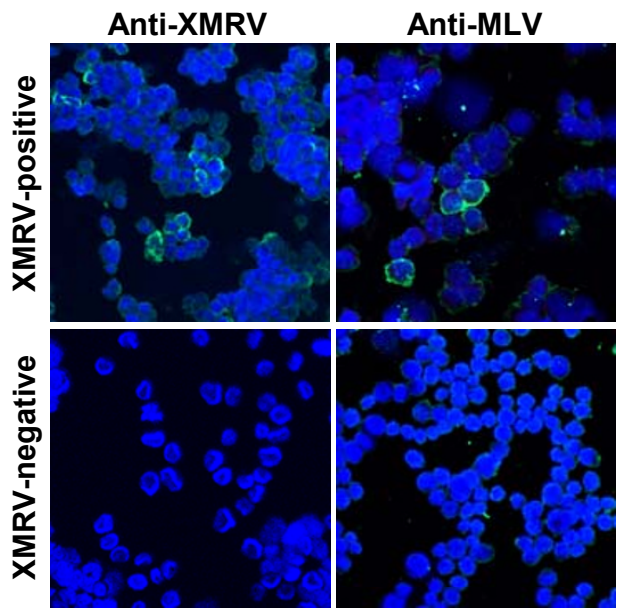

B

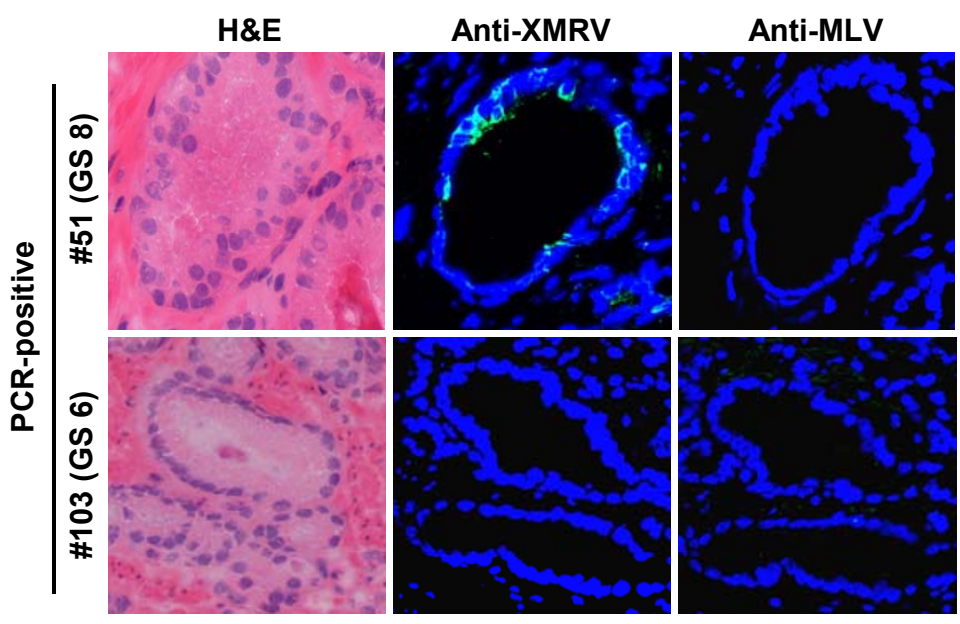

C
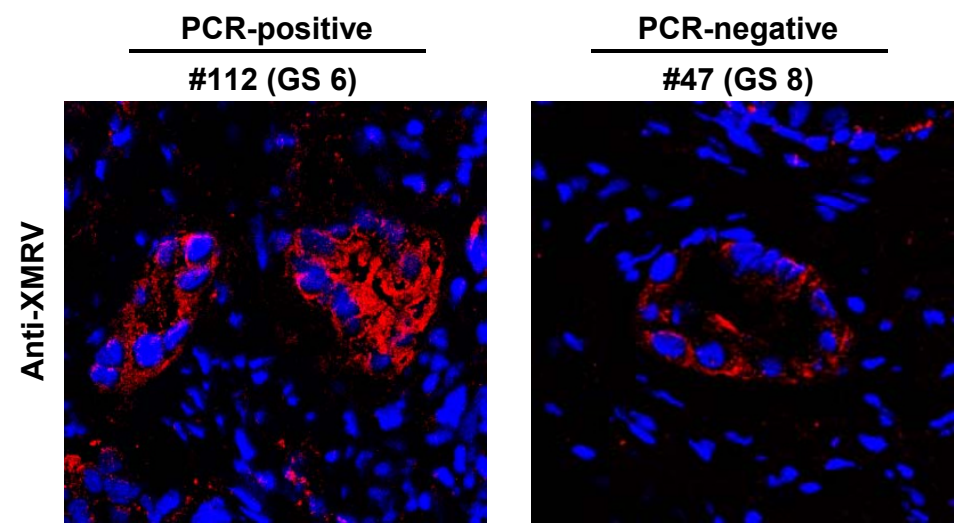

Figure 3 Detection of XMRV in prostate cancer tissues. (A) Specificity of anti-XMRV antiserum and anti-MLV antibody. 293T cells transfected with XMRV infectious plasmid (pcDNA3.1(-)/NP62) were stained with either rabbit anti-XMRV or goat anti-MLV. No positive staining was observed in control uninfected 293T cells. (B) Serial tissue sections from qPCR positive samples, including \#51 (Gleason score (GS) 8) and \#103 (GS 6) were immunostained with either anti-XMRV or anti-MLV antibody. H\&E staining from each sample is also shown. (C) Serial tissue sections from qPCR positive (\#112, GS 6) and negative (\#47, GS 8) samples were immunostained with anti-MLV antibody, followed by TexasRed-conjugated donkey anti-rabbit antibody (Jackson ImmunoResearch Laboratories, Inc., 1:200). 


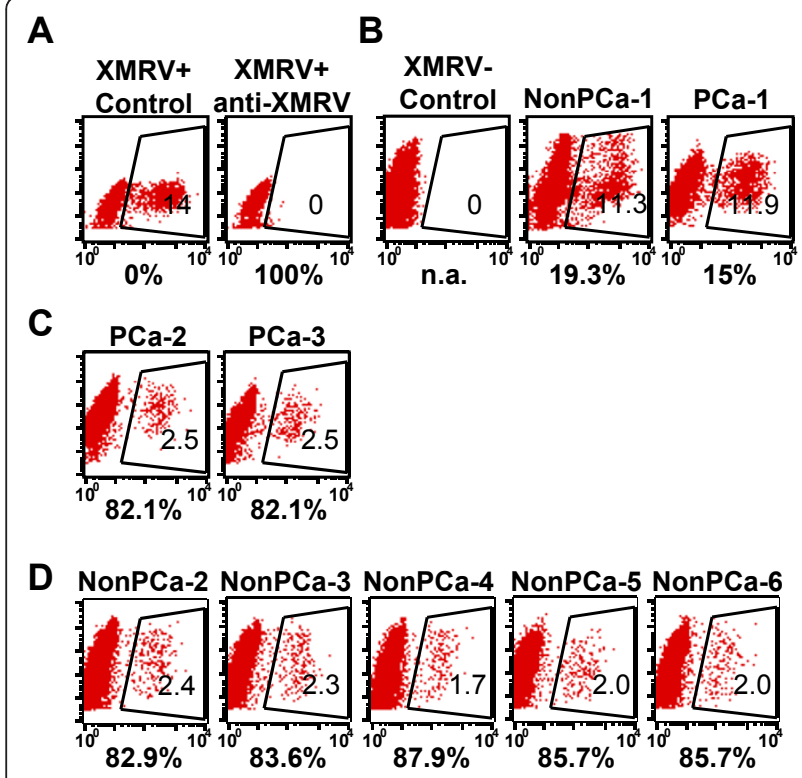

Figure 4 Neutralization activity of patient sera. (A) XMRV infected 293T cells (XMRV+ control) and XMRV-infected and treated with anti-XMRV sera at a dilution of 1:10 [14] are shown. (B) Data from non-XMRV infected 293T cells is shown as a control. Patients samples which did not show positive neutralization reaction (Patients with non-prostate cancer (NonPCa)-1, Patients with prostate cancer (PCa)-1) are shown. (C) Two samples that showed positive reaction from patients with prostate cancer (PCa-2 and -3) are shown. (D) Six samples that showed positive neutralization reaction from patients with non-prostate cancer (NonPCa-2 to -6) are shown. 1:10 dilution of sera were applied for all the experiments. Percent GFP positive and percent neutralization are indicated within the gated areas and below the flow data, respectively. The percent neutralization was calculated as the reciprocal of infectivity, with a maximum infectivity being determined by incubation of the virus with an uninfected mouse serum. n.a., not applicable.

Unfortunately, we were not successful, likely due to low viral copy numbers in the clinical samples. Very recently, Robinson et al. [23] and Oakes et al. [22] reported similar observations; all XMRV PCR-positive specimens contained detectable levels of mouse mitochondrial or endogenous retroelements (IAPs). Together with our data, these findings highlight the difficulty of avoiding DNA contamination in clinical samples and the risk of testing contaminated samples as XMRV-positive by sensitive PCR detection assays. As a possible source of contamination, Sato et al. [24] demonstrated that a commercially available hot-start PCR enzyme contained mouse DNA. We used several enzymes and obtained similar results. Thus, it is unlikely that the contaminating mouse genome originated from a PCR kit. Since we could amplify the viral sequences from multiple aliquoted DNA samples, they appeared to be contaminated before or during the DNA isolation step, most likely during tissue sectioning on a microtome.

XMRV antigen-positive cells have been detected in prostatic stromal fibroblasts [3] or in malignant prostatic epithelium [7]. Our IHC study using two different antisera showed conflicting results. The goat anti-MLV antibody found no viral antigens in clinical samples, while the rabbit anti-XMRV antibody used in the study by Schlaberg, Singh and colleagues [7] detected antigenpositive cells in prostatic epithelium. Strikingly, the goat anti-MLV serum did not stain the cells, which were IHC-positive by the anti-XMRV rabbit serum, in serial sections of the same tissue. The rabbit antiserum also found antigen-positive cells in PCR-negative sections, confirming the observations of Schlaberg and colleagues who reported frequent detection of IHC-positive samples in PCR-negative tissues [7]. Importantly, both the rabbit and goat antibodies detected XMRV in experimentally infected cells with high sensitivity (Figure 3 ). Together, these observations strongly suggest that the rabbit antiserum is detecting a non-viral antigen sporadically expressed by tumor cells in the tissue section. We conclude that our PCa samples do not have XMRV antigen-expressing cells that are detectable by IHC.

We recently reported that Mus pahari mice elicit potent XMRV-specific humoral immune response upon XMRV infection [14]. At a serum dilution of 1:640, antisera from infected animals almost completely blocked XMRV infection [14]. Similarly, an animal study using XMRV-infected rhesus macaques and sensitive ELISA detection assays showed that infected animals rapidly develop antibodies against XMRV proteins, including gp70 (Env), p15E (transmembrane), and p30 (CA) [27]. These results indicate that XMRV is strongly immunogenic in these animals. In contrast, we were unable to detect strong XMRV-specific neutralizing antibodies in our 360 patients, age 50-70, with or without PCa. This observation further suggests a lack of XMRV in our cohorts. It is possible, although less likely, that XMRV is not immunogenic in humans or that XMRV-specific immune response might have disappeared in these relatively elderly patients.

\section{Conclusion}

In our study population of patients with or without $\mathrm{PCa}$ from the USA, we found no evidence of infection with XMRV using PCR, IHC and serological tests. Our negative results are in accordance with previous studies using sensitive PCR, ELISA and Western blot assays, which failed to detect PCR or seropositive samples in a large number of blood donors, HTLV- and HIV-infected, or patients with or without CFS [9-12,21,27-31]. Our results indicate the possible false-positive detection of XMRV/ MLV-related sequences or antigen-positive cells through 
laboratory contamination or non-specific immune reaction respectively, and underscore the need for careful validation of previous and future studies.

\section{Materials and methods}

Prostate tissues and plasma samples from patients

Prostate tissues and plasma samples were obtained from Mayo Clinic Biospecimen Core with an approval from the Institutional Review Boards. Frozen sections of prostate cancer tissues $(10 \mu \mathrm{m})$ were identified as 1 through 150 in duplicates. These samples included 40 normal/ low grade Gleason score, 70 intermediate (Gleason score 5-7), and 40 high grade (Gleason score 8-10) with men aged between 50-70 years old. For plasma analysis, total of 360 plasma samples from 50-70 year old male patients including 159 prostate patients (Gleason score 5-7) and 201 patients with no prostate cancer or urological disorders were used in this study.

\section{TaqMan qPCR}

Total cellular DNA was extracted by PureLink Genomic DNA Mini Kit according to the manufacturer's protocol (Invitrogen). All samples were eluted in $50 \mu \mathrm{l}$ of elution buffer, and the concentration and quality of the DNA were determined by a NanoDrop Spectrophotometer. For the real-time PCR assay, TaqMan Universal PCR Master Mix (Roche) was used along with $2 \mu$ of each sample. Primers were used at a range of $230 \mathrm{nM}$ to $300 \mathrm{nM}$ final concentration. TaqMan probe \#51 from Roche Universal Probe Library was used for XMRV-gag at $100 \mathrm{nM}$ final concentration. A standard curve was created by using serially diluted XMRV plasmid (pcDNA3.1 (-)/VP62). The assay was analyzed by the ABI 7300 RealTime PCR System using the default thermal cycling conditions for the two-step RT-PCR method and FAM reporter [13].

\section{Genotyping}

RNASEL genotype was determined by nested PCR amplification using outer primers 5'-CTGGGGTTCTATGAGAAGCAAG-3' and 5'-TGAGCTTTCAGATCCTC AAATG-3', and inner primers 5'-GAGAGAACAGTCACTTGGTGAC-3' and 5'-CAGCCCACTTGATGCTC TTATC-3' with $p f x$ polymerase (Invitrogen). Final PCR products were purified with QIAquick PCR Purification Kit (Qiagen) before sequence analysis.

\section{Neutralization assay}

The neutralization assay was carried out using GFPencoding XMRV as described previously [13,14]. Briefly, 293T cells were transfected with pcDNA3.1(-)/VP62 and a GFP-encoding retroviral vector using FuGene 6 (Roche). Serum samples were heat inactivated at $56^{\circ} \mathrm{C}$ for $30 \mathrm{~min}$. A mixture of plasma samples and $2.5 \times 10^{4}$ infectious units of GFP-carrying XMRV were incubated at $37^{\circ} \mathrm{C}$ for $30 \mathrm{~min}$ before infecting $293 \mathrm{~T}$ cells $\left(5 \times 10^{4}\right)$. Three days post-infection, cells were resuspended, fixed with $4 \%$ paraformaldehyde and analyzed by flow cytometry (BD FACScan). The percentages of GFP-positive cells were measured using CellQuestPro software [14].

\section{Western blot analysis}

For Western blot analysis of XMRV proteins, cell lysates of prostate cancer (PC-3) cell line (ATCC) and PC-3 infected with XMRV were harvested in $1.0 \mathrm{ml}$ of RIPA lysis buffer. Cell debris was removed by centrifugation, and the supernatant was diluted with Laemmli sample buffer containing $\beta$-mercaptoethanol. After heatdenaturation at $95^{\circ} \mathrm{C}$ for $5 \mathrm{~min}, 10 \mu \mathrm{l}$ of proteins were subjected to SDS-PAGE with a $4-15 \%$ gradient gel (Bio-Rad), and transferred to a polyvinylidene diflouride membrane at $0.7 \mathrm{~mA} / \mathrm{cm}^{2}$ for $40 \mathrm{~min}$. Membranes were blocked in $5 \%$ milk/PBS, then stained with patient's plasma samples diluted to 1:250, followed by anti-human IgG (1:1000, Jackson ImmunoResearch Laboratories, Inc.).

\section{Immuno-histochemistry}

Immunohistochemistry was performed on tissue samples from patients with or without prostate cancer. Sections were fixed with $4 \%$ paraformaldehyde for $20 \mathrm{~min}$ and treated with $0.3 \%$ Triton X100 for $15 \mathrm{~min}$ at room temperature. They were then blocked with 5\% FBS/PBS for 30 min and immunostained with rabbit-anti XMRV (kindly provided by Dr. Ila Singh, University of Utah) or goat-anti p30/gp70 (NCI HD625 CAT No. 04-0109, LOT No. 81S000262, Quality Biotech, kindly provided by Dr. Yasuhiro Takeuchi, UCL) at a dilution of 1:500 for $4 \mathrm{~h}$ at room temperature. FITC-conjugated anti-rabbit IgG (1:500; Amersham) or DyLight 488-conjugated anti-goat IgG (1:500; Jackson ImmunoResearch Lab) were applied for $2 \mathrm{~h}$ at room temperature. Nuclei were then counter-stained with 4'-6-Diamidino-2-phenylindole (DAPI), and analyzed by confocal microscopy (Zeiss).

\section{Nested PCR and sequence analysis of proviral DNA}

Sequence analysis was performed as previously described [14]. Briefly, DNA was extracted by PureLink Genomic DNA Mini Kit (Invitrogen). Nested-PCR was performed for XMRV gag (primers for outer gag: 5'-ACGAGTT CGTATTCCCGGCCGCA-3' and 5'-CCGCCTCTTCT TCATTGTTC-3', primers for inner gag: 5'-GCCCATT CTGTATCAGTTAA-3' and 5'-AGAGGGTAAGGGCAGGGTAA-3') with platinum Taq polymerase (Cat. no. 10966-034, Invitrogen). The resulting PCR products from a total of 4 patient samples $\# 15$, \#51, \#52 and \#112) were cloned into the TOPO vector (Invitrogen). Sequences from the two patient samples \#53 and \#103 
were not analyzed. From patients \#15, 51, 52, 112, we sequenced 2, 2, 4, 1 clones, and got 2, 2, 2, 1 different sequences, respectively. They were analyzed by DNADynamo (BlueTractorSoftware).

\section{Phylogenetic analyses}

Seven unique gag gene sequences (255 to $528 \mathrm{nt}$ ), amplified from our clinical samples (GenBank no. JF288878, JF288879, JF288880, JF288881, JF288882, JF288883, and JF288884), were manually aligned with previously described murine leukemia virus gag gene sequences ( $\mathrm{n}=79)$, 22Rv1 cell line derived gag sequences (1605 nt; $\mathrm{n}=15)$, XMRV gag sequences apparently amplified from prostate cancer and CFS samples $(\mathrm{n}=7)[6]$, as well as $6 \mathrm{MLV}$ virus gag sequences isolated from chronic fatigue syndrome samples [16]. Bayesian phylogenies were reconstructed as previously described [6]. The Markov chain Monte Carlo search was set to 10,000,000 iterations, with trees sampled every 1000th generation, and with a $20 \%$ burn in. The phylogeny of the aforementioned sequences was also reconstructed by maximum likelihood (ML) inference under the general time reversible model of nucleotide substitution, with gamma-distributed rate heterogeneity and proportion of invariable sites, using the program RAxML (data not shown) [32]. The ML topology was assessed by neighbor joining bootstrapping with 1000 replicates using the program PAUP*.

\section{A semi-nested mouse-specific mtDNA PCR}

We used a PCR assay for mouse mitochondrial DNA reported to be able to detect $2.5 \mathrm{fg}$ of mouse DNA in the presence of $35 \mathrm{ng}$ human background DNA [16]. Using this assay, we tested whether our samples were contaminated with mouse DNA. DNA from PCR positive samples were PCR amplified with KOD Hot Start DNA Polymerase following the manufactures instruction (Novagen) as described [16]. The resulting PCR fragments were further cloned into the TOPO vector and the sequences were confirmed to be identical to the mouse cytochrome b gene by DNA BLAST.

\footnotetext{
Acknowledgements

Rabbit-anti XMRV and goat-anti p30/gp70 were kindly provided by Dr. Ila Singh and Dr. Yasuhiro Takeuchi respectively. This work was supported by the National Institute of Health (Al093186), Mayo Clinic Career Development Project in Prostate SPORE grant CA91956-080013, the Mayo Foundation (YI) Wellcome Trust senior fellowship WT090940 (GJT) European Community's Seventh Framework Programme (FP7/2007-2013) under the project 'Collaborative HIV and Anti-HIV Drug Resistance Network (CHAIN)', grant agreement no. 223131 (SH) and the National Institute of Health Research UCL/UCLH Comprehensive Biomedical Research Centre (GJT).
}

\section{Author details}

${ }^{1}$ Department of Molecular Medicine, Mayo Clinic, Rochester, MN 55905 USA. ${ }^{2}$ Department of Infection and Immunity, MRC Centre for Medical Molecular Virology, University College London, 46 Cleveland St, London W1T 4JF, UK.

\section{Authors' contributions}

TS, SH, KAS, JMT, and PRB performed experiments. TS, SH, GT and YI designed the experiments, analyzed the data and wrote the paper. All authors read and approved the final manuscript.

\section{Competing interests}

The authors declare that they have no competing interests.

Received: 2 February 2011 Accepted: 29 March 2011

Published: 29 March 2011

\section{References}

1. Simard J, Dumont M, Soucy P, Labrie F: Perspective: prostate cancer susceptibility genes. Endocrinology 2002, 143:2029-2040.

2. Casey G, Neville PJ, Plummer SJ, Xiang Y, Krumroy LM, Klein EA, Catalona WJ, Nupponen N, Carpten JD, Trent JM, et al: RNASEL Arg462Gln variant is implicated in up to $13 \%$ of prostate cancer cases. Nat Genet 2002, 32:581-583.

3. Urisman A, Molinaro RJ, Fischer N, Plummer SJ, Casey G, Klein EA, Malathi K Magi-Galluzzi C, Tubbs RR, Ganem D, et al: Identification of a novel Gammaretrovirus in prostate tumors of patients homozygous for R462Q RNASEL variant. PLoS Pathog 2006, 2:e25.

4. Dong B, Kim S, Hong S, Das Gupta J, Malathi K, Klein EA, Ganem D, Derisi JL, Chow SA, Silverman RH: An infectious retrovirus susceptible to an IFN antiviral pathway from human prostate tumors. Proc Natl Acad Sci USA 2007, 104:1655-1660.

5. Baliji S, Liu Q, Kozak CA: Common inbred strains of the laboratory mouse that are susceptible to infection by mouse xenotropic gammaretroviruses and the human-derived retrovirus XMRV. J Virol 84:12841-12849.

6. Hue S, Gray ER, Gall A, Katzourakis A, Tan CP, Houldcroft CJ, McLaren S, Pillay D, Futreal A, Garson JA, et al: Disease-associated XMRV sequences are consistent with laboratory contamination. Retrovirology 2010, 7:111.

7. Schlaberg R, Choe DJ, Brown KR, Thaker HM, Singh IR: XMRV is present in malignant prostatic epithelium and is associated with prostate cancer, especially high-grade tumors. Proc Natl Acad Sci USA 2009, 106:16351-16356.

8. Arnold RS, Makarova NV, Osunkoya AO, Suppiah S, Scott TA, Johnson NA, Bhosle SM, Liotta D, Hunter E, Marshall FF, et al: XMRV infection in patients with prostate cancer: novel serologic assay and correlation with PCR and FISH. Urology 2010, 75:755-761.

9. Aloia AL, Sfanos KS, Isaacs WB, Zheng Q, Maldarelli F, De Marzo AM, Rein A: XMRV: a new virus in prostate cancer? Cancer Res 2010, 70:10028-10033.

10. Verhaegh GW, de Jong AS, Smit FP, Jannink SA, Melchers WJ, Schalken JA Prevalence of human xenotropic murine leukemia virus-related gammaretrovirus (XMRV) in dutch prostate cancer patients. Prostate 2010, 71(4):415-20, Epub 2010 Sep 28.

11. Hohn $O$, Krause H, Barbarotto P, Niederstadt L, Beimforde N, Denner J, Miller K, Kurth R, Bannert N: Lack of evidence for xenotropic murine leukemia virus-related virus(XMRV) in German prostate cancer patients. Retrovirology 2009, 6:92.

12. Fischer N, Hellwinkel O, Schulz C, Chun FK, Huland H, Aepfelbacher M, Schlomm T: Prevalence of human gammaretrovirus XMRV in sporadic prostate cancer. J Clin Virol 2008, 43:277-283.

13. Sakuma $R$, Sakuma $T$, Ohmine $S$, Silverman $R H$, Ikeda $Y$ : Xenotropic murine leukemia virus-related virus is susceptible to AZT. Virology 2010, 397:1-6.

14. Sakuma T, Tonne JM, Squillace KA, Ohmine S, Thatava T, Peng KW, Barry MA, Ikeda Y: Early events in retrovirus XMRV infection of the wildderived mouse Mus pahari. J Virol 2011, 85:1205-1213.

15. Lombardi VC, Ruscetti FW, Das Gupta J, Pfost MA, Hagen KS, Peterson DL, Ruscetti SK, Bagni RK, Petrow-Sadowski C, Gold B, et al: Detection of an infectious retrovirus, XMRV, in blood cells of patients with chronic fatigue syndrome. Science 2009, 326:585-589.

16. Lo SC, Pripuzova N, Li B, Komaroff AL, Hung GC, Wang R, Alter H]: Detection of MLV-related virus gene sequences in blood of patients with chronic fatigue syndrome and healthy blood donors. Proc Natl Acad Sci USA 2010, 107:15874-15879.

17. Stamatakis A: RAxML-VI-HPC: maximum likelihood-based phylogenetic analyses with thousands of taxa and mixed models. Bioinformatics 2006 22:2688-2690. 
18. Malet I, Belnard M, Agut H, Cahour A: From RNA to quasispecies: a DNA polymerase with proofreading activity is highly recommended for accurate assessment of viral diversity. J Virol Methods 2003, 109:161-170.

19. Bracho MA, Moya A, Barrio E: Contribution of Taq polymerase-induced errors to the estimation of RNA virus diversity. J Gen Virol 1998, 79(Pt 12):2921-2928.

20. Cline J, Braman JC, Hogrefe HH: PCR fidelity of pfu DNA polymerase and other thermostable DNA polymerases. Nucleic Acids Res 1996, 24:3546-3551.

21. Switzer WM, Jia H, Hohn O, Zheng H, Tang S, Shankar A, Bannert N, Simmons G, Hendry RM, Falkenberg VR, et al: Absence of evidence of xenotropic murine leukemia virus-related virus infection in persons with chronic fatigue syndrome and healthy controls in the United States. Retrovirology 2010, 7:57.

22. Oakes B, Tai AK, Cingoz O, Henefield MH, Levine S, Coffin JM, Huber BT: Contamination of human DNA samples with mouse DNA can lead to false detection of XMRV-like sequences. Retrovirology 2010, 7:109.

23. Robinson MJ, Erlwein OW, Kaye S, Weber J, Cingoz O, Patel A, Walker MM, Kim WJ, Uiprasertkul M, Coffin JM, McClure MO: Mouse DNA contamination in human tissue tested for XMRV. Retrovirology 2010, 7:108.

24. Sato E, Furuta RA, Miyazawa T: An Endogenous Murine Leukemia Viral Genome Contaminant in a Commercial RT-PCR Kit is Amplified Using Standard Primers for XMRV. Retrovirology 2010, 7:110.

25. Kim S, Kim N, Dong B, Boren D, Lee SA, Das Gupta J, Gaughan C, Klein EA Lee C, Silverman RH, Chow SA: Integration site preference of xenotropic murine leukemia virus-related virus, a new human retrovirus associated with prostate cancer. J Virol 2008, 82:9964-9977.

26. Tomonaga K, Coffin JM: Structures of endogenous nonecotropic murine leukemia virus (MLV) long terminal repeats in wild mice: implication for evolution of MLVs. J Virol 1999, 73:4327-4340.

27. Qiu X, Swanson P, Luk KC, Tu B, Villinger F, Das Gupta J, Silverman RH, Klein EA, Devare S, Schochetman G, Hackett I Jr: Characterization of antibodies elicited by XMRV infection and development of immunoassays useful for epidemiologic studies. Retrovirology 2010, 7:68,

28. Groom HC, Boucherit VC, Makinson K, Randal E, Baptista S, Hagan S, Gow JW, Mattes FM, Breuer J, Kerr JR, et al: Absence of xenotropic murine leukaemia virus-related virus in UK patients with chronic fatigue syndrome. Retrovirology 2010, 7:10

29. Hong P, Li J, Li Y: Failure to detect Xenotropic murine leukaemia virusrelated virus in Chinese patients with chronic fatigue syndrome. Virol J 2010, 7:224

30. Hohn O, Strohschein K, Brandt AU, Seeher S, Klein S, Kurth R, Paul F, Meisel C, Scheibenbogen C, Bannert N: No evidence for XMRV in German CFS and MS patients with fatigue despite the ability of the virus to infect human blood cells in vitro. PLoS One 2010, 5:e15632.

31. Erlwein O, Kaye S, McClure MO, Weber J, Wills G, Collier D, Wessely S, Cleare A: Failure to detect the novel retrovirus XMRV in chronic fatigue syndrome. PLoS One 2010, 5:e8519.

32. Swofford DL: PAUP*. Phylogenetic analysis using parsimony (* and other methods). Sinauer Associates, Sunderland, MA; 19984, versions.

doi:10.1186/1742-4690-8-23

Cite this article as: Sakuma et al.: No evidence of XMRV in prostate cancer cohorts in the Midwestern United States. Retrovirology 2011 8:23.

\section{Submit your next manuscript to BioMed Central and take full advantage of:}

- Convenient online submission

- Thorough peer review

- No space constraints or color figure charges

- Immediate publication on acceptance

- Inclusion in PubMed, CAS, Scopus and Google Scholar

- Research which is freely available for redistribution

Submit your manuscript at www.biomedcentral.com/submit
Biomed Central 